\title{
Measurement of Ramp Rate Sensitivity in Model Dipoles With Ebanol-Coated Cable
}

\author{
C. Haddock*, V. Kovachev*, and D. Capone* \\ Superconducting Super Collider Laboratory ${ }^{\dagger}$ \\ 2275 North Highway 77, Waxahachie, Texas
}

\begin{abstract}
Ramp rate sensitivity in superconducting dipole magnets has been shown to vary in an unpredictable fashion between magnets constructed using similar fabrication techniques. Test results from a series of model magnets are presented, one of which was constructed using ebanolcoated strands. The ramp rate sensitivity and ac loss observed is described.
\end{abstract}

\section{INTRODUCTION}

In order to determine the training, ramp rate and magnetic field performance of prototype dipoles intended for the SSC, a comprehensive program of cold magnet testing was performed at Fermi and Brookhaven National Laboratories [1]. The results showed two distinct families of quench current versus current ramp rate, as shown in figure 1. The first family (known as type "A") showed an initial nearly flat quench current up to about $20 \mathrm{~A} / \mathrm{s}$, then an almost linear decrease of quench current with ramp rate. The linear portion of the curve, however, varies significantly amongst members of this family.

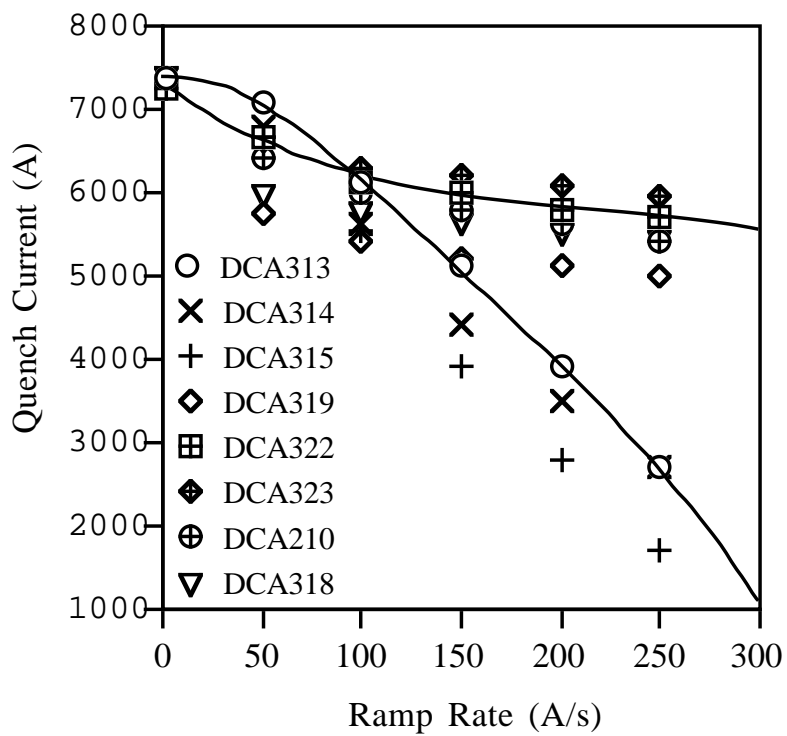

Figure 1: Quench current dependence on ramp rate for $50 \mathrm{~mm}$ aperture $15 \mathrm{~m}$ length dipole magnets.

*Present address: Texas National Research Laboratory Commission, 2275 North Highway 77, Suite 100, Waxahachie, Texas 75165

$\dagger$ Operated by Universities Research Assocation under contract with the Department of Energy.
The second family (type "B") showed a rapid initial decrease in quench current at low ramp rates followed by a much slower decrease at high ramp rates. The curves shown in the figure are not intended to fit all data sets, but rather to illustrate the two trends. The $50 \mathrm{~mm}$ short model magnets have shown similar behavior but somewhat less pronounced.

The collider ring of the SSC was to have been filled with beam from a smaller synchrotron known as the High Energy Booster (HEB) whose dipole magnets were intended to utilize essentially the same design as those of the collider. The HEB was to accelerate beam up to $2 \mathrm{TeV}$ for injection into the collider, at which point the peak current in the dipole magnets would be $6600 \mathrm{~A}$. In order to achieve this acceleration the ramp rate of current in the dipoles was to be $62 \mathrm{~A} / \mathrm{s}$. It can be seen from figure 1 that some dipoles exhibiting type B behavior would quench before reaching the peak current planned for HEB operation, while some of those exhibiting type A behavior would reach the required current with inadequate current margin.

\section{RAMP RATE STUDIES}

In order to study this problem further, a program was initiated between the SSC and Westinghouse Corporation (WEC), the designated contractor for the HEB dipole manufacture. A considerable enhancement in the understanding of ramp rate behavior has resulted $[2,3,4]$.

The combination of cable twist pitch and interstrand resistance produces trapezoidal loops within the Rutherford Cable [4]; ramping the magnet produces eddy currents within these loops. These currents flow through the interstrand resistance generating heating, and superpose to the transport current of one side of the cable. The eddy current distribution within the cable depends upon both the "crossover" resistance of overlapping strands as well as the parallel resistance of side-byside strands. Furthermore, these resistance values vary along the magnet length as strand surface conditions and interface pressure values change. The combined effect is to produce eddy current induced heating and local enhancement of the transport current, both of which effectively reduce the margin of the conductor.

For type A ramp rate sensitivity it is believed that the interstrand heating dominates. The resistance between strands can be as low as $1 \mu \Omega$ or less and interstrand heating is uniformly distributed throughout the cable. Eddy current heating within the cable may therefore be fit to the magnet dependence observed.

Type B behavior is less well understood. It is believed that for this behavior, crossover resistance for most of the coil is 
relatively high, resulting in loops of large inductance along the cable, which are finally closed by a less frequent low value of interstrand resistance [5]. The result is a greater sensitivity at lower values of ramp rate.

\section{A. Model Magnet Studies}

Short versions of the full-length designs allow cost effective and timely testing of most features of full-length magnets. A short dipole program was begun to assess the performance of the WEC dipole design. The magnet cross section consisted of a two-layer $\cos \theta$ design with inner and outer coil sections connected via a ramp splice internal to the coil. The NbTi cable consisted of 30-strand, $0.81 \mathrm{~mm}$ diameter 1.3:1 copper to superconductor ratio for the inner coil and 36-strand, 0.67 $\mathrm{mm}$ diameter 1.8:1 copper to superconductor ratio for the outer coil. The cable twist pitch was $88 \mathrm{~mm}$. Single strand crossover resistance for the uncoated cable was expected to be of the order of 1 or $10 \mu \Omega$ based on studies of sectioning of similar magnets [6], while the interstrand resistance of the ebanol-coated cables was expected to be much larger, of the order of $10 \mathrm{~m} \Omega$.

The quench performance of the cross section was quite acceptable, with typically two training quenches to plateau. Ramp rate performance of the first two magnets of the series, which had untreated cable, was type A.

\section{EBANOL-COATED CONDUCTOR FABRICATION}

The practice of insulating adjacent strands or every other strand for the purposes of reducing ramp rate sensitivity is not new; it was first pursued at Fermi National Laboratory while building the Tevatron. That cable was composed of alternating strands with ebanol and stabrite coatings, respectively. Ebanol is a commercial copper-oxide-based coating material which converts a copper surface to a robust copper-oxide coating. The stabrite coating is a silver-tin solder coating. This cable configuration is commonly referred to as "zebra" because of the alternating black and "white" appearance.

For this study, two cable configurations were prepared in order to determine the resulting effects of the coatings on the ramp-rate-dependent quenching of short SSC dipole magnets. The first is identified as "panther" cable (every strand coated with ebanol) and the second is identified as "tiger" cable (alternate strands were left uncoated). The magnet mechanical design performed sufficiently well so that changes to improve training were unnecessary. Thus, the comparisons in ramp rate performance due to cable coating alone could be evaluated. Unfortunately, funding was terminated for the SSC project during the program and only the results for the panther versus bare cable can be presented here.

Strands of Inner wire, taken from a single production unit, were used for this set of magnets. Sufficient wire for four complete sets of Inner coils was used. Approximately twothirds of the wire was coated with ebanol using a commercial dipping process. The strands were uniformly coated with ebanol upon return from the vendor. The wires had a high degree of debris on the strands due to deposition of the ebanol onto the spools during the processing. The wires were thoroughly cleaned with alcohol wipes on the respooling line prior to the fabrication of the cables.

Bare copper strands were spliced onto the front of each of the coated strands as a leader which was used to set up the appropriate cable parameters prior to fabricating the actual cables. This turned out to be ineffective, since the surface friction of the ebanol-coated strands is sufficiently different as to require a new set of cabling machine parameters to produce the cables with the coated strands. The set-up for both the panther and tiger cables was quite similar but were very different from cables made with bare copper strands.

\section{TEST RESULTS}

The ramp rate sensitivity measured on the ebanol-coated cable magnet, known as DSB703, is shown in figure 2. The first two magnets produced in the series, which have no coating on the cable, are also shown for comparison.

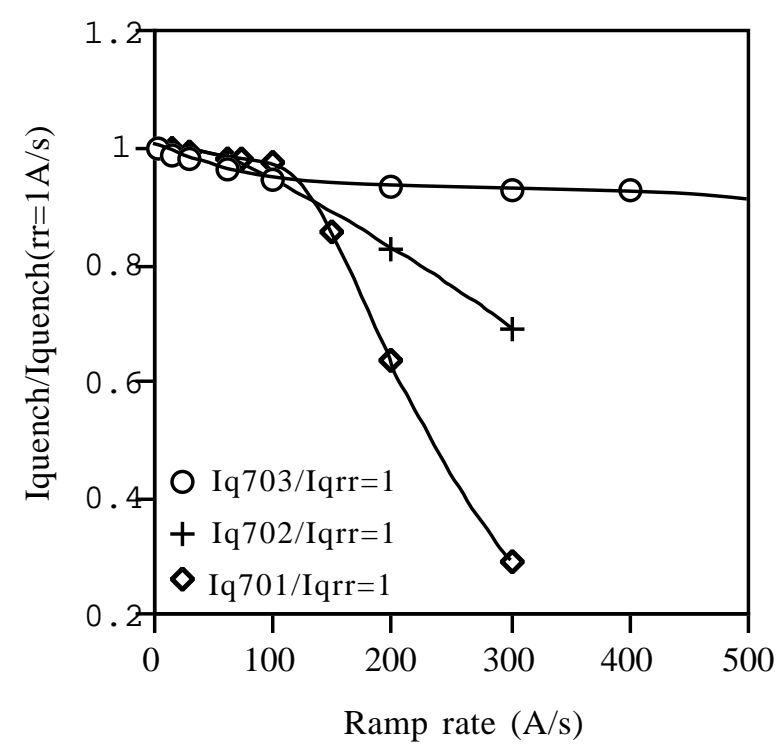

Figure 2: Quench current dependence on ramp rate for ebanolcoated and uncoated cable magnets.

The plateau (field limited) currents for DSB701,2 and 3 are $7758 \mathrm{~A}, 8058 \mathrm{~A}$ and $7914 \mathrm{~A}$, respectively. For comparison of ramp rate performance, figure 2 shows the quench currents as fractions of the plateau current. The plateau currents were measured at low ramp rates $\sim 1 \mathrm{~A} / \mathrm{s}$ where ramp rate induced heating effects can be neglected.

AC losses measured on the uncoated and coated cable magnets are shown in figure 3 . Eddy current losses have been reduced from $1.15 \mathrm{~J} / \mathrm{m} /$ cycle $/(\mathrm{A} / \mathrm{s})$ to $0.58 \mathrm{~J} / \mathrm{m} / \mathrm{cycle} /(\mathrm{A} / \mathrm{s})$. Bipolar losses are shown, as the magnet was intended for bipolar use.

The hysteresis losses remain the same as expected and provide a convenient verification of the two measurements. 


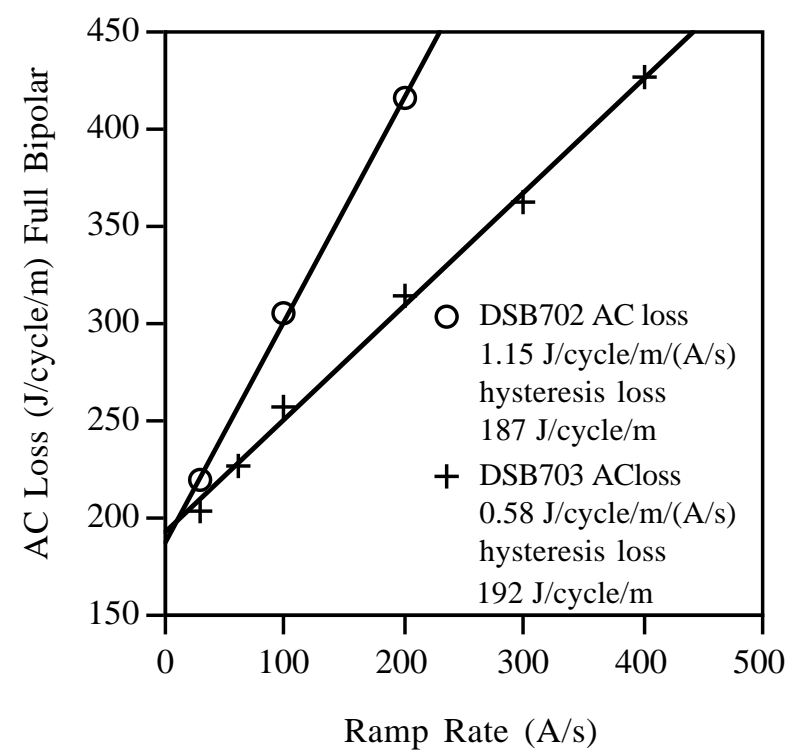

Figure 3: AC loss data for ebanol-coated and uncoated cable magnets.

\section{A. Discussion}

It can be seen that the ebanol-coated cable has a type B behavior, which was somewhat unexpected. This may be explained by mechanical instability of the ebanol insulation, which during cable fabrication and coil winding results in small areas of bare cable and low interstrand resistance. Although ramp rate sensitivity at the higher ramp rates has been significantly reduced, at the lower rates one has the situation we wished to avoid. It would appear that the solution is to have a uniformly distributed crossover interstrand resistance in order to induce type A behavior, but to keep the value high enough such that extreme behavior (somewhat shown by DSB701) is avoided. This may be accomplished by coating the cable with a different material which has a relatively high resistivitiy and yet does not wear off the strand during the cable and magnet fabrication processes, resulting in the type B behavior observed.

\section{CONCLUSIONS}

A program of short magnet fabrication has allowed us to compare the ramp rate performance of a magnet design where only the coating on the magnet cable was changed. Coated cables show promise for the control of ramp rate sensitivity and ac losses.

\section{REFERENCES}

[1] J. Tompkins et al, "Summaries of Iquench vs Ramp Rate Studies on Full length 50 mm Dipole Magnets" SSC Internal Publication MD-TA-241 January 1992.

[2] V. T. Kovachev, M. J. Neal, D. W. Capone II, and W. J. Carr Jr., "Interstrand Resistance of SSC Magnets" Cryogenics 34 Number 101994 pp 813-820.

[3] G. T. Mallick Jr., W. J. Carr Jr., J. M. Toms, V. T. Kovachev, "Eddy Currents in Superconducting Rutherford Cables" Accepted for publication in Cryogenics.

[4] W. J. Carr Jr., and V. T. Kovachev, "Interstrand Eddy Current Losses in SSC Magnets". Accepted for publication in Cryogenics.

[5] W. J. Carr Jr., G. T. Mallick Jr., and V. T. Kovachev, "Theory of the Quench Current of B type SSC Magnets". Westinghouse STC Internal report, Pittsburgh Pennsylvania September 30, 1994.

[6] V. T. Kovachev, M. J. Neal, J. Seuntjens, J. Madison, S. Graham, P. Cline, D. W. Capone II, and M. Wake, "Interstrand Resistance of Selected Sections of DCA312". SSC Internal Publication SSCL-651 November 1993. 